\title{
A Cross-Sectional Examination of Extrapyramidal Side-Effects (EPSE) in a Specialist Palliative Medicine Inpatient Unit

\section{Background:}

$>$ EPSE are serious side effects caused by anti-psychotics and other medications

A core principle of palliative care involves optimising quality of life

$>$ If side-effects of medications are burdensome it is imperative that we address this issue

$>$ There are a number of ways in which EPSE present or manifest, namely, dystonia, pseudo-parkinsonism, akathesia and tardive dyskinesia

$>$ EPSE are not routinely screened for in hospice settings

$>$ Barnes Akathesia Rating Scale (BARS) is a rating scale for drug-induced akathesia ${ }^{1}$

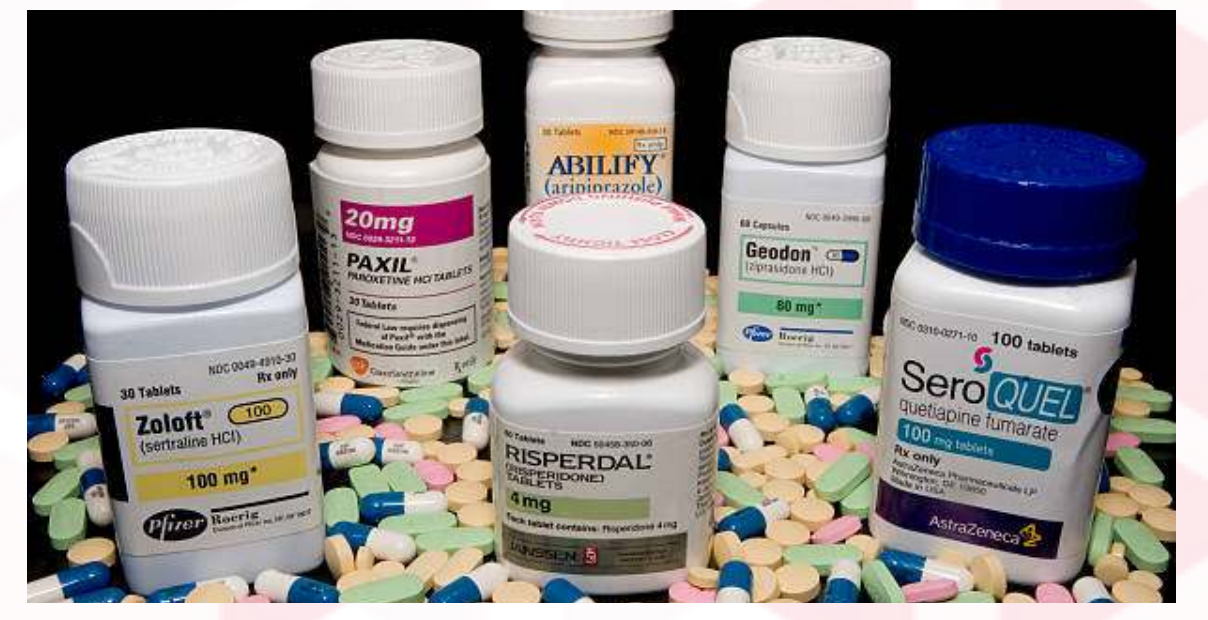

$>$ The Modified Simpson-Angus Scale (MSAS) is used in rating pseudo-parkinsonism and allows for a "not rateable scale" if one is unable to assess an element of the scale ${ }^{2}$

\section{Aims}

$>$ To determine and describe the burden of extrapyramidal side-effects (EPSE) in a specialist inpatient unit

\section{Methods:}
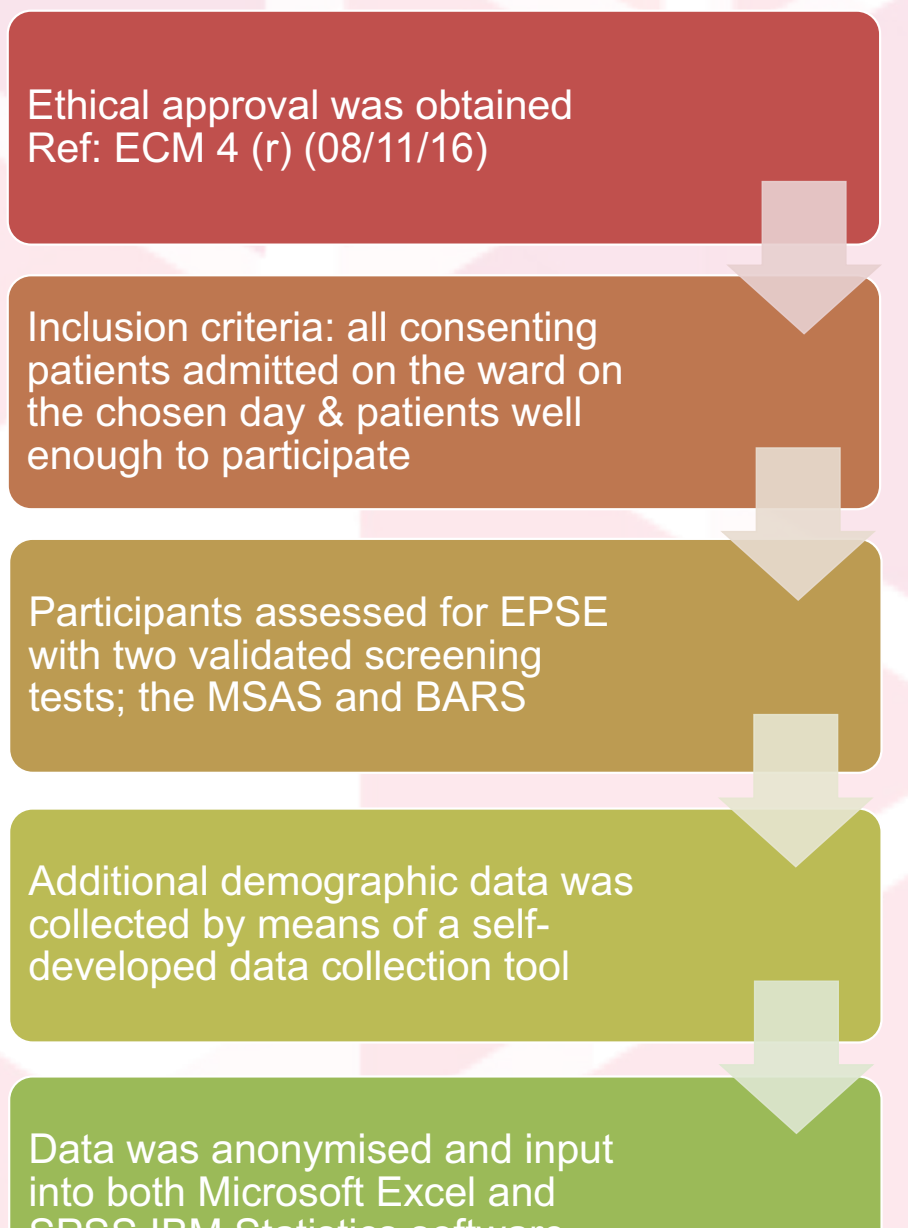

\section{Results:}

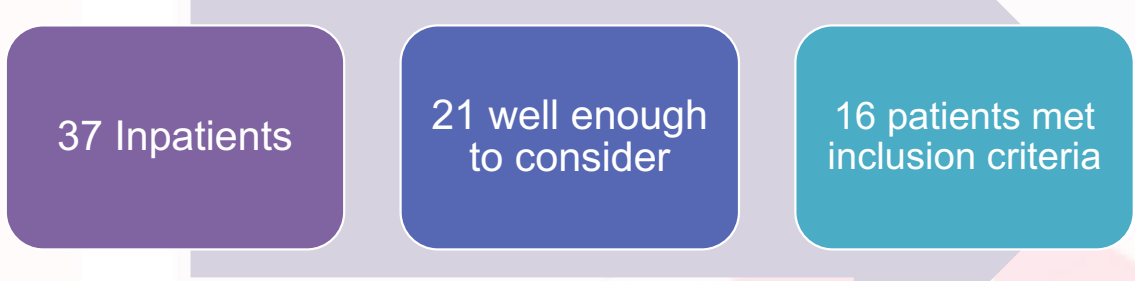

$>$ There were 8 male $(50 \%)$ and 8 female $(50 \%)$ participants with a mean age of 72 years

$>$ Median length of admission was 32 days

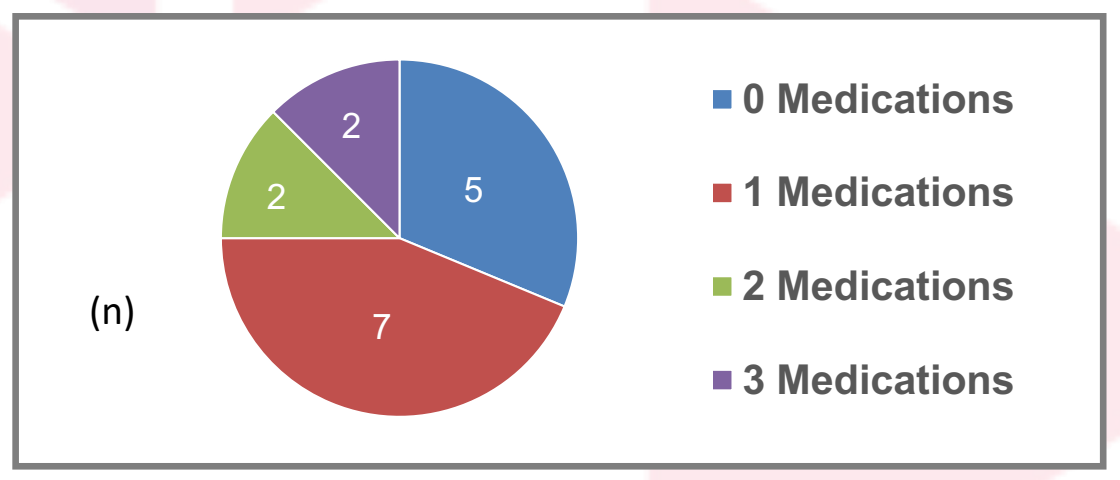

Figure 1. No. of patients taking regular medications with potentia to cause EPSE

$>$ Nine regular medications associated with EPSE were identified ${ }^{3}$ - amitriptyline,

haloperidol, olanzapine, levomepromazine, metoclopramide, fluoxetine, escitalopram, diazepam and bromazepam

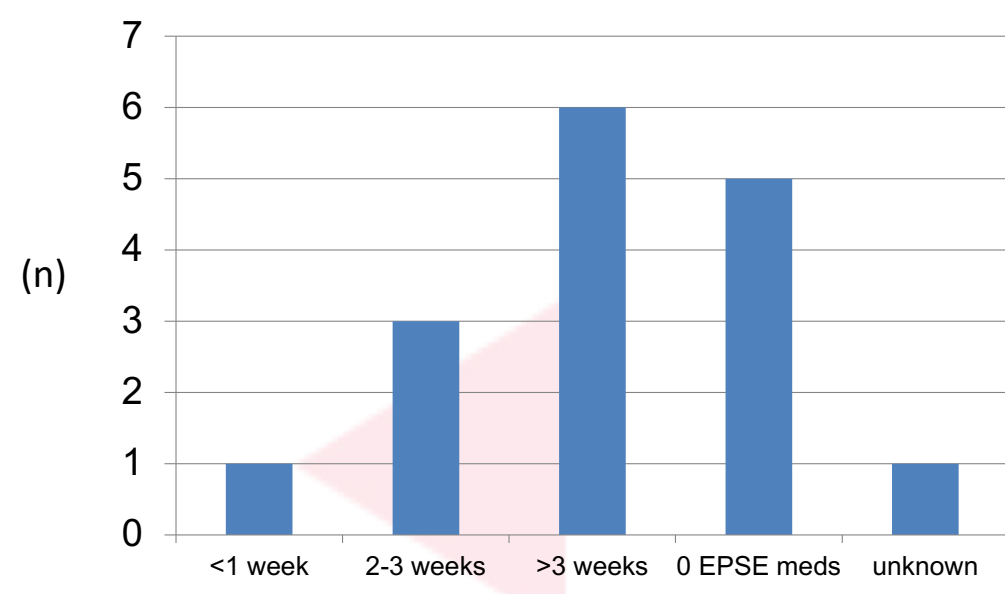

Figure 2. MSAS and duration of treatment with medications associated with EPSE ( $n=$ number of medications)

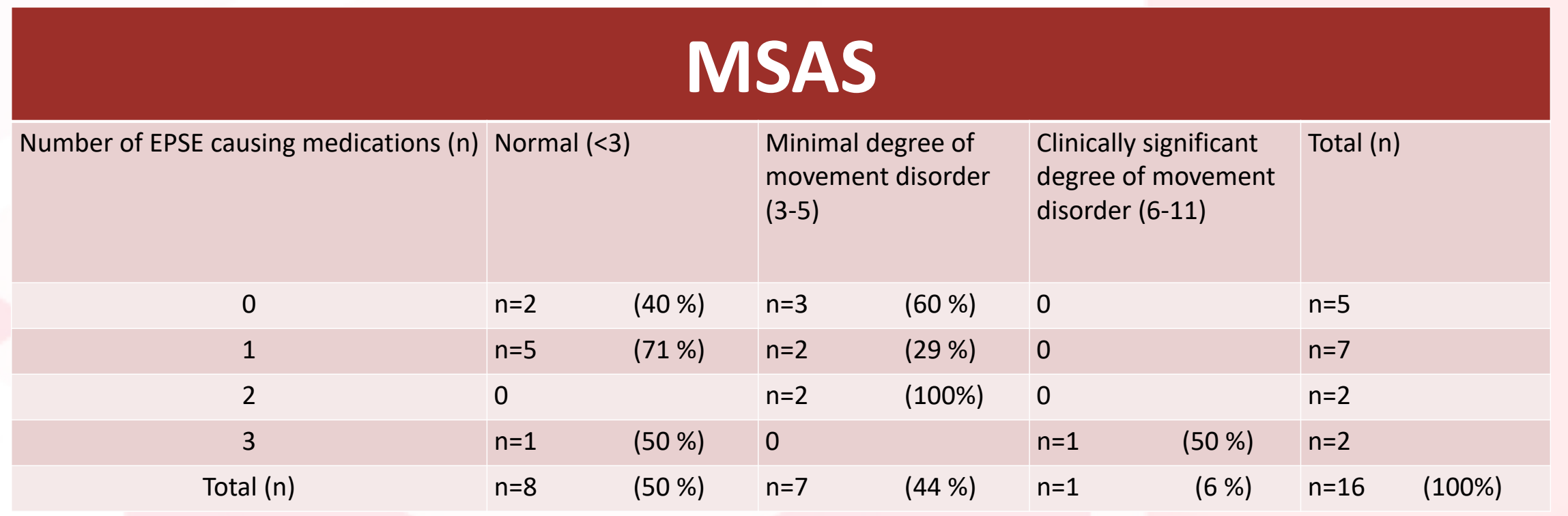

Table 1. MSAS scores and number of medications associated with EPSE

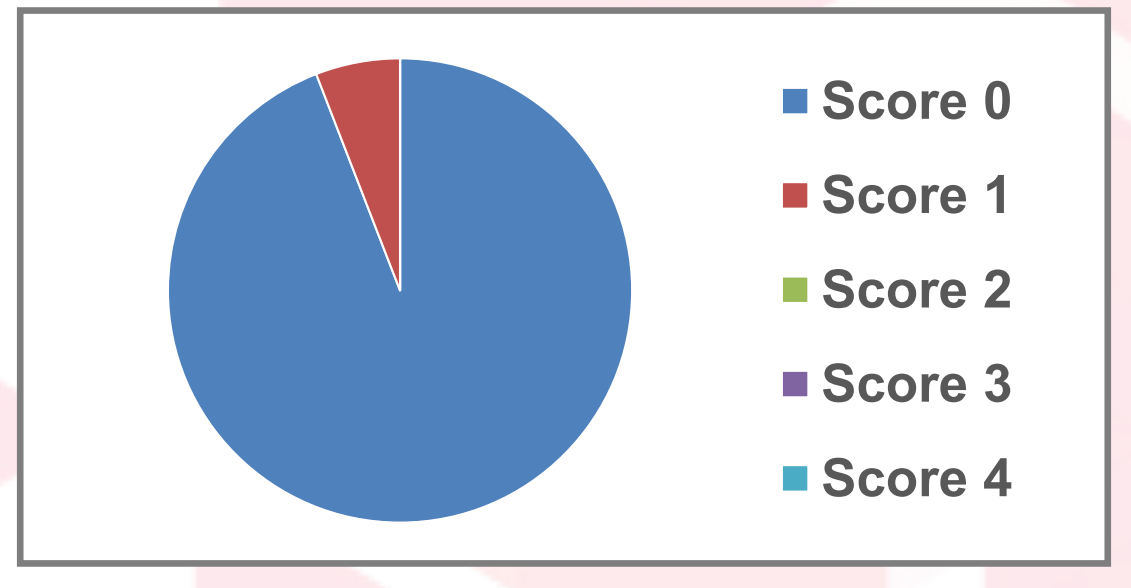

Figure 3. Barnes Akathesia Rating Scale Scores

$>$ One patient $(6 \%)$ scored $1 / 5$ in BARS

$>$ It was appropriate to examine 2 (12.5\%) patients both seated and standing

$>$ Risk factors - 6 (38\%) patients had one, 3 $(19 \%)$ had 2 and 1 patient $(6 \%)$ had 3 risk factors for EPSE

$>3$ patients with 1 risk factor and 2 patients with 2 risk factors scored 3-5 with MSAS

$>$ The patient with 3 risk factors scored 6-11 on the MSAS

$>$ The remaining 4 patients scored $<3$

\section{$\mathbb{T}$ Strengths \& Limitations:}

$>$ To our knowledge this is the first study to examine EPSE in a palliative medicine inpatient population

> The data was examined using bivariate and multivariate analysis; however, due to small numbers it was not possible to run inferential statistics

\section{Conclusion:}

$>50 \%$ screened positive for EPSE

$>$ The complete BARS was unsuitable for most participants $(87.5 \%)$

$>$ The MSAS while allowing a not-rateable score may underestimate EPSE

$>$ The frailty of an inpatient unit population impacts on applicability of screening tools and may therefore underestimate the burden of the problem in this population

Development of a population-specific screening tool warrants further investigation 